\title{
On the mean square of the error term for an extended Selberg class
}

by

Anne De Roton (Nancy)

1. Notations. We write, as usual, $s=\sigma+i \tau$ and $\bar{f}(s)=\overline{f(\bar{s})}$. We recall here the definition of the extended Selberg class $S^{\#}$ studied by J. Kaczorowski and A. Perelli in a series of papers (see [KP0] for an introduction). The class $S^{\#}$ consists of the non-identically vanishing functions $F(s)$ satisfying the following conditions:

(i) for $\sigma>1, F(s)$ is an absolutely convergent Dirichlet series

$$
F(s)=\sum_{n=1}^{\infty} a_{n} n^{-s} ;
$$

(ii) for some integer $m \geq 0,(s-1)^{m} F(s)$ is an entire function of finite order;

(iii) $F(s)$ satisfies a functional equation of the form

$$
\Phi(s)=\epsilon \bar{\Phi}(1-s), \quad \text { where } \quad \Phi(s)=Q^{s} \gamma(s) F(s),
$$

with

$$
\gamma(s)=\prod_{j=1}^{r} \Gamma\left(\lambda_{j} s+\mu_{j}\right),
$$

where $Q>0,|\epsilon|=1$, and for all $j \in[1, r], \lambda_{j}>0, \mu_{j} \in \mathbb{C}$ with $\Re \mu_{j} \geq 0$.

The Selberg class $S$ is the set of functions $F \in S^{\#}$ satisfying two more axioms:

- (Ramanujan hypothesis) for every $\varepsilon>0, a_{n} \ll n^{\varepsilon}$;

- (Euler product) for sufficiently large $\sigma$,

$$
\log F(s)=\sum_{n=1}^{\infty} \frac{b_{n}}{n^{s}}
$$

2000 Mathematics Subject Classification: Primary 11M41. 
where $b_{n}=0$ unless $n$ is a positive prime power, and $b_{n} \ll n^{\theta}$ for some $\theta<1 / 2$.

We define the class $S^{\# b}$ as the set of functions $F \in S^{\#}$ satisfying furthermore the following axiom:

- for every $\varepsilon>0$ and every $x \geq 1, \sum_{n \leq x}\left|a_{n}\right|^{2}=O_{\varepsilon}\left(x^{1+\varepsilon}\right)$.

Notation. The degree $d$ of $F \in S^{\#}$ is defined as $d=2 \sum_{j=1}^{r} \lambda_{j}$, and the polar order $m_{F}$ of $F$ is the least integer $m$ satisfying (ii). We also write

$$
\xi=2 \sum_{j=1}^{r}\left(\mu_{j}-\frac{1}{2}\right) \quad \text { and } \beta=\prod_{j=1}^{r} \lambda_{j}^{-2 \lambda_{j}} .
$$

REMARK 1.1. The functional equation (1) may be written in the form

$$
F(1-s)=\epsilon Q^{2 s-1} \frac{\bar{\gamma}(s)}{\gamma(1-s)} \bar{F}(s) .
$$

We define $S(x)$ as the sum of the residues of the function $F(s) x^{s} / s$, and the error term $E(x)$ as

$$
E(x)=\sum_{n \leq x}^{\prime} a_{n}-S(x),
$$

the prime indicating that the last term has to be multiplied by $1 / 2$ if $x=n$. In case $m_{F} \neq 0, E(x)$ is the error term for the summatory function of the coefficients of the Dirichlet series $F(x)$.

REMARK 1.2. Since $s=1$ is the only singularity of the function $F$, we can write $S(x)$ in the form

$$
S(x)=F(0)+\operatorname{Res}\left(\frac{F(s)}{s} x^{s}, 1\right)=F(0)+x P_{F}(\log x),
$$

where $P_{F}$ is a polynomial function of degree $m_{F}-1$. The last equality results from a simple argument; the reader may consult [Lan, Vorbemerkung über $R(x)$, p. 697] for more details.

2. Introduction and statement of the results. In this paper we give pointwise and mean square upper bounds for the error term $E(x)$ associated with a function $F$ in the extended Selberg class $S^{\#}$.

Estimates for $E(x)$ and $\int_{1}^{x}|E(y)|^{2} d y$ were first obtained, in the special case of the divisor problem, by Voronoï in 1904 and Cramér in 1922 respectively (see $[\mathrm{V}]$ and $[\mathrm{Cr}]$ ). Since then, this has been generalised to larger classes of Dirichlet series.

As early as 1912, Landau give an estimate of $E(x)$ for a class of Dirichlet series satisfying a functional equation of general type involving multiple gamma factors. His method of proof is based on an application of the Perron 
formula and the residue theorem. A kind of van der Corput method is also used.

In 1964, Chandrasekharan and Narasimhan gave an estimate for $E(x)$ and $\int_{1}^{x}|E(y)|^{2} d y$ for functions of general type (see [CN1]-[CN3], [Ch]). Their method is based on the study of some hypergeometric functions. Since then, their results have been improved for functions of high degree by Redmond in [R1] and [R2].

Nevertheless, as far as we know, no estimate has been given in the case of the extended Selberg class. The large classes already studied in the literature do not comprise the class $S^{\#}$ since, in the functional equations that have been considered, either the $\mu_{j}$ have to be real, or no conjugate occurs.

Our results for a function in $S^{\#}$ are identical to those obtained by Landau, Chandrasekharan and Narasimhan in the case where the $\mu_{j}$ are real. We prove the following theorems.

TheOREM 2.1. Let $F \in S^{\#}$ be a function of degree $d \geq 2$ and let $\varepsilon>0$. Then, for $x \geq 1$, we have

$$
E(x)=O\left(x^{(d-1) /(d+1)+\varepsilon}\right) .
$$

REMARK 2.1. The conclusion of Theorem 2.1 holds true for functions $F$ of degree $d<2$ in $S^{\# b}$. The additional Ramanujan hypothesis is most probably redundant but we have not managed to avoid it. Nevertheless, we notice that:

- from the explicit description of the functions in $S^{\#}$ of degree $d \leq 1$ given by Kaczorowski and Perelli in [KP1], we easily deduce that the conclusion of Theorem 2.1 holds true for them;

- the degree of the functions of $S^{\#}$ is conjecturally a positive integer (see $[\mathrm{KP} 0]$ ), and Kaczorowski and Perelli have already proved that there is no function in $S^{\#}$ of degree $1<d<5 / 3$ (see [KP2]).

Theorem 2.1 for functions $F$ of degree $d<2$ in $S^{\# b}$ is therefore either well known or conjecturally empty. Nevertheless, we prove the result announced in this remark later, since the case $5 / 3<d<2$ has to be treated independently from the degree conjecture.

Theorem 2.2. Assume that $F \in S^{\# b}$ and let $\varepsilon>0$. Then, for $x \geq 1$,

$$
\int_{1}^{x}|E(y)|^{2} d y= \begin{cases}O\left(x^{2-1 / d}\right) & \text { if } 0<d<3, \\ O\left(x^{3-4 / d+\varepsilon}\right) & \text { if } d \geq 3 .\end{cases}
$$

The Selberg class was introduced in [S] and since then it has been studied in various papers by Conrey and Gosh (see [CG1], [CG2]), Murty (see [M]), Kaczorowski and Perelli (see $[\mathrm{KP} 0]-[\mathrm{KP} 2], \ldots)$. The structure of the extended Selberg class $S^{\#}$ we are considering here has been particularly stud- 
ied by the last two authors. In [KP1] and [KP2], they investigate some incomplete Fox hypergeometric functions and establish a link between these functions and those of $S^{\#}$.

Many conjectures have been devised about $S$ and $S^{\#}$. Especially, it is expected that any function $F \in S$ satisfies the generalised Lindelöf hypothesis:

Conjecture 2.1 .

$$
\forall \varepsilon>0, \quad F(1 / 2+i \tau) \ll_{\varepsilon} \tau^{\varepsilon} .
$$

This problem is also still open for a function in $S^{\#}$ (see [CG2]). This conjecture is strongly connected with the study of the error term $E(x)$ since we can prove that a function $F$ in $S$ satisfies the generalised Lindelöf hypothesis if and only if

$$
\frac{F^{k}(1 / 2+i \tau)}{1 / 2+i \tau} \in L^{2}(\mathbb{R}) \quad(\forall k \in \mathbb{N})
$$

and we prove in [dR2] that if $F \in S^{\#}$, then

$$
\frac{F(1 / 2+i \tau)}{1 / 2+i \tau} \in L^{2}(\mathbb{R}) \Leftrightarrow E \in L^{2}\left(\left[0, \infty\left[, d x / x^{2}\right),\right.\right.
$$

where $E$ is the error term associated to $F$.

Our results imply that $E \in L^{2}\left(\left[0, \infty\left[, d x / x^{2}\right)\right.\right.$ for functions in $S^{\#}$ of degree less than 4 . This result is of great relevance in [dR2].

To prove Theorem 2.1, we follow Landau's method in [Lan]. A few arguments have to be modified. The methods of proof of Theorem 2.2 both involve an identity relating a smoothed version of the error term to a series of hypergeometric functions. We then study these hypergeometric functions and expansions. To return to the initial function, we use finite differences. This method was used by Chandrasekharan and Narasimhan in [CN1] and [CN2], except for the study of the hypergeometric function which was easier in their case. Here, we have to connect our hypergeometric functions to Bessel functions to obtain some results similar to theirs.

We start by giving some technical lemmas. In Section 3, we draw up a list of consequences of the complex Stirling formula and in particular we give some vertical strip estimates for a function in the Selberg class. In Section 4, we give some technical lemmas. Section 5 will be devoted to the study of Bessel functions. In Section 6, we connect a smoothed version of the error term with a hypergeometric function studied in Section 7. In Sections 8 and 9, we prove Theorems 2.1 and 2.2 respectively.

The author would like to thank the referee for his very fruitful comments, for pointing out several inaccuracies and for suggesting how to improve our results, especially in Section 8. We would also like to thank Professor J. Ka- 
czorowski for having suggested the use of Bessel functions during a very fruitful conversation in Cetraro.

3. Some consequences of the Stirling formula. We give here some technical estimates coming from the following Stirling formula. The reader will find a demonstration of this result in [Bo, formule (19), $\S$ VII.2.3].

Proposition 3.1. There exist constants $c_{\nu}=c_{\nu}(a)$ such that, for all $M \in \mathbb{N}$, as $|s| \rightarrow \infty$,

$$
\begin{aligned}
& \log \Gamma(s+a) \\
& \quad=\left(s+a-\frac{1}{2}\right) \log s-s+\frac{1}{2} \log 2 \pi+\sum_{\nu=1}^{M} c_{\nu} s^{-\nu}+O\left(\frac{1}{|s|^{M+1}}\right)
\end{aligned}
$$

uniformly for $|\arg (s)| \leq \pi-\varepsilon$ with a fixed $\varepsilon>0$ and a in a compact subset of $\mathbb{C}$.

3.1. Study of a function coming from the functional equation

Lemma 3.1. Let $\gamma(s)$ be as in (2). Then, uniformly for $s \in \mathbb{C}$ such that $\varepsilon \leq|\arg s| \leq \pi-\varepsilon$, for all $M \in \mathbb{N}$, as $|s| \rightarrow \infty$,

$$
\begin{aligned}
\log \left(\frac{\bar{\gamma}(s)}{\gamma(1-s)}\right)= & \frac{d}{2} s(\log s+\log (-s))-s(\log \beta+d) \\
& +\frac{1}{2} \bar{\xi} \log s-\frac{1}{2}(\xi+d) \log (-s) \\
& +\frac{1}{2} \log \beta+2 i \sum_{j=1}^{r} \Im \mu_{j} \log \lambda_{j}+\sum_{\nu=1}^{M} c_{\nu} s^{-\nu}+O\left(|s|^{-M-1}\right) .
\end{aligned}
$$

This follows easily from the Stirling formula.

Lemma 3.2. Let $\gamma(s)$ be as in (2). Then, uniformly for $\sigma_{1} \leq \sigma \leq \sigma_{2}$, as $|\tau| \rightarrow \infty$

$$
\frac{\bar{\gamma}(s)}{\gamma(1-s)}=c(\sigma, \tau)|\tau|^{d(\sigma-1 / 2)} e^{i(d \tau \log |\tau|-(\log \beta+d) \tau-\Im \xi \log |\tau|)}(1+O(1 /|\tau|))
$$

where $c(\sigma, \tau) \in \mathbb{C}$ only depends on $\sigma$ and the sign of $\tau$.

Notation. For $a>0$ and $\nu \in \mathbb{R}$, we define

$$
f(s)=f_{a, \nu}(s):=\frac{1}{2}\left(\frac{2}{a}\right)^{s-\nu} \frac{\Gamma(s / 2)}{\Gamma(\nu-s / 2+1)} .
$$

We shall establish a link between the function $f$ and the multiple gamma factor $\gamma$.

Lemma 3.3. For $\alpha>0, a>0, \nu \in \mathbb{R}, \kappa \in \mathbb{C}$, there exist real numbers $K=K(\alpha, a, \kappa, \nu)$ and $c_{j}^{\prime}=c_{j}^{\prime}(\alpha, a, \kappa, \nu), j \in \mathbb{N}$, such that for all $M \in \mathbb{N}$, 
as $|s| \rightarrow \infty$,

$$
\begin{aligned}
\log (f(\alpha s+\kappa)) & \\
= & \frac{\alpha}{2} s(\log s+\log (-s))+\alpha(\ln \alpha-\ln a-1) s+\frac{\kappa-1}{2} \log s \\
& +\left(\frac{\kappa-1}{2}-\nu\right) \log (-s)+K+\sum_{j=1}^{M} c_{j}^{\prime} s^{-j}+O\left(\frac{1}{|s|^{M+1}}\right)
\end{aligned}
$$

uniformly for s such that $\varepsilon<|\arg s|<\pi-\varepsilon$ with $\varepsilon>0$.

In particular,

$$
|f(s)| \asymp|\tau|^{\sigma-1-\nu} \quad \text { as }|\tau| \rightarrow \infty,
$$

uniformly for $\sigma \in\left[\sigma_{1}, \sigma_{2}\right]$.

Definition 3.1. For $\varrho \in \mathbb{Z}, \varrho \geq-1$, we define

$$
G_{\varrho}(s)=\frac{\Gamma(1-s) \bar{\gamma}(s)}{\Gamma(2+\varrho-s) \gamma(1-s)} .
$$

By Stirling's formula, there is a complex sequence $c_{\nu}^{(1)}=c_{\nu}^{(1)}\left(\lambda_{1}, \ldots, \lambda_{r}\right.$; $\left.\mu_{1}, \ldots, \mu_{r} ; \varrho\right)$ such that for all $M \in \mathbb{N}^{*}$, as $|s| \rightarrow \infty$

$$
\text { (7) } \begin{aligned}
\log G_{\varrho}(s)= & \frac{d}{2} s(\log s+\log (-s))+s\left(\sum_{j=1}^{r} 2 \lambda_{j} \log \lambda_{j}-d\right) \\
& +\frac{1}{2} \bar{\xi} \log s-\left(\frac{1}{2}(\xi+d)+\varrho+1\right) \log (-s) \\
& +\left(\sum_{j=1}^{r}\left(-\lambda_{j}-2 i \Im \mu_{j}\right) \log \lambda_{j}\right)+\sum_{\nu=1}^{M} c_{\nu}^{(1)} s^{-\nu}+O\left(\frac{1}{|s|^{M+1}}\right)
\end{aligned}
$$

uniformly for $\varepsilon \leq|\arg (s)| \leq \pi-\varepsilon$ with $\varepsilon>0$.

In particular,

$$
\left|G_{\varrho}(s)\right| \asymp|\tau|^{d(\sigma-1 / 2)-\varrho-1} \quad \text { as }|\tau| \rightarrow \infty,
$$

uniformly for $\sigma \in\left[\sigma_{1}, \sigma_{2}\right]$.

Notation. We write

$$
\alpha=d, \quad \kappa=1+\bar{\xi}, \quad \nu=1+\Re \xi+d / 2+\varrho, \quad a=d \beta^{1 / d} .
$$

Comparing (7) to (5), we show that there exist real numbers $e_{k}=$ $e_{k}\left(\lambda_{1}, \ldots, \lambda_{r} ; \mu_{1}, \ldots, \mu_{r} ; \varrho\right)$ such that, as $|\tau| \rightarrow \infty$,

$r_{m}(s):=G_{\varrho}(s)-\sum_{k=0}^{m} F_{k}(s)=O\left(|f(\alpha s+\kappa)||s|^{-m-1}\right)=O\left(|\tau|^{d(\sigma-1 / 2)-\varrho-m-2}\right)$ 
uniformly for $\sigma \in\left[\sigma_{1}, \sigma_{2}\right]$, with

$$
F_{k}(s)=e_{k} f(\alpha s+\kappa) \frac{(-1)^{k}}{(\alpha s+\kappa-k) \cdots(\alpha s+\kappa-1)} .
$$

3.2. Estimates for the value of a quotient of $\Gamma$ functions in half integers

Proposition 3.2. For $\nu \in \mathbb{R}, \Re z=N+1 / 2$ with $N \in \mathbb{N}$ and $N \geq$ $|\nu|+1 / 2$, we have

$$
\frac{\Gamma(1-z)}{\Gamma(\nu+z)} \ll \frac{e^{2 N}}{|z|^{N}|\nu+z|^{N+\nu}} .
$$

Proof. By the complement formula, we get

$$
\frac{\Gamma(1-z)}{\Gamma(\nu+z)}=\frac{\pi / \sin (\pi z)}{\Gamma(\nu+z) \Gamma(z)} .
$$

For $z=N+1 / 2+i t$, we have

$$
\left|\frac{\pi}{\sin (\pi z)}\right|=\left|\frac{2 i \pi}{e^{i \pi(N+1 / 2)} e^{-\pi t}-e^{-i \pi(N+1 / 2)} e^{\pi t}}\right|=\frac{2 \pi}{e^{-\pi|t|}+e^{\pi|t|}} .
$$

On the other hand, by (4), we have uniformly for $\Re z \geq|\nu|+1$,

$$
\begin{aligned}
\log |\Gamma(\nu+z) \Gamma(z)|= & (\Re z-1 / 2) \log |z|+(\nu+\Re z-1 / 2) \log |\nu+z|-|\Im z| \pi \\
& +\Im z\left(\arctan \frac{\Re z}{\Im z}+\arctan \frac{\Re z+\nu}{\Im z}\right) \\
& -2 \Re z-\nu+\log 2 \pi+O(1 /|z|) \\
\geq & (\Re z-1 / 2) \log |z|+(\nu+\Re z-1 / 2) \log |\nu+z| \\
& -|\Im z| \pi-2 \Re z-\nu+\log 2 \pi+O(1 /|z|) .
\end{aligned}
$$

For $N \geq|\nu|+1 / 2$ and $z=N+1 / 2+i t$, we get

$$
\begin{aligned}
\log |\Gamma(\nu+z) \Gamma(z)| \geq & N \log |z|+(\nu+N) \log |\nu+z| \\
& -|t| \pi-2 N-1-\nu+\log 2 \pi+O(1 /|z|) .
\end{aligned}
$$

So we have

$$
\frac{\Gamma(1-z)}{\Gamma(\nu+z)} \ll|z|^{-N}|\nu+z|^{-\nu-N} e^{2 N}
$$

3.3. Estimates of a function of the Selberg class in some vertical strips LEMmA 3.4. Let $F$ be a function in the extended Selberg class $S^{\#}$.

- Let $\varepsilon>0$. Then $|F(s)|=O_{\varepsilon}(1)$ uniformly for $\sigma \geq 1+\varepsilon$ and $\tau \in \mathbb{R}$.

- For $\sigma<0, F(s)=O_{\sigma}\left((1+|\tau|)^{d(1 / 2-\sigma)}\right)$.

- For all $\varepsilon>0$, if $-\varepsilon \leq \sigma \leq 1+\varepsilon$, then $F(s)=O_{\sigma, \varepsilon}\left((1+|\tau|)^{\frac{d}{2}(1-\sigma+\varepsilon)}\right)$.

Proof. The first estimate comes from the fact that $F(s)$ is an absolutely convergent Dirichlet series for $\sigma>1$. The functional equation and 
Lemma 3.2 provide the second one. To prove the last estimate, we use a Phagmén-Lindelöf argument.

\section{Some more lemmas}

Definition 4.1. If $T>0$ and $\Lambda, c \in \mathbb{R}$ with $c \leq \Lambda$, we define the path

$$
C(T, c, \Lambda):=] c-i \infty ; c-i T ; \Lambda-i T ; \Lambda+i T ; c+i T ; c+i \infty[.
$$

In order to move paths of integration, we shall use the following lemma.

LEMMA 4.1. Let $f$ be a meromorphic function on $\mathbb{C}$ such that:

- $T_{0}:=\sup \{|\Im \varrho|: \varrho$ a pole of $f\}<\infty$,

- $\Lambda_{0}:=\sup \{\Re \varrho: \varrho$ a pole of $f\}<\infty$,

- there exist $a>0$ and $b \in \mathbb{R}$ such that, for all $\sigma_{1}<\sigma_{2}$, we have, uniformly in $\sigma \in\left[\sigma_{1}, \sigma_{2}\right],|f(s)| \ll|\tau|^{a \sigma-b-1}$ as $|\tau| \rightarrow \infty$.

If

$$
T>T_{0}, \quad c<b / a, \quad \Lambda>\Lambda_{0},
$$

then, for $x>0$, the integral

$$
I(x):=\frac{1}{2 i \pi} \int_{C(T, c, \Lambda)} f(s) x^{-s} d s
$$

is convergent and its value does not depend on $T, c$ and $\Lambda$ satisfying (10). Furthermore, for all $\varepsilon>0$, we have $I(x)=O_{\varepsilon}\left(x^{-b / a+\varepsilon}\right)$ as $x \rightarrow \infty$.

Proof. Under the assumptions of the lemma, the integrals are convergent on the path $C(T, c, \Lambda)$. To get the full conclusion, it is enough to use the residue theorem between the two paths $C(T, c, \Lambda)$ and $C\left(T^{\prime}, c^{\prime}, \Lambda^{\prime}\right)$ with $T, c, \Lambda$ and $T^{\prime}, c^{\prime}, \Lambda^{\prime}$ satisfying (10) and observe that the horizontal integrals tend to zero.

LEMMA 4.2. Let $a>0$ and $b \geq 1$. Then

$$
\int_{0}^{\infty} \frac{d \tau}{\left(a^{2}+\tau^{2}\right)^{b}} \leq \frac{\pi}{2} \frac{a^{1-2 b}}{\sqrt{b}} .
$$

Proof. Since $a>0$ and $b>1 / 2$, the integral is convergent. Furthermore,

$$
\int_{0}^{\infty} \frac{d \tau}{\left(a^{2}+\tau^{2}\right)^{b}}=a^{1-2 b} \int_{0}^{\infty} \frac{d \tau}{\left(1+\tau^{2}\right)^{b}} .
$$

By MAPLE, we get

$$
\int_{0}^{\infty} \frac{d \tau}{\left(1+\tau^{2}\right)^{b}}=\frac{\sqrt{\pi}}{2} \frac{\Gamma(b-1 / 2)}{\Gamma(b)} .
$$


The logarithmic derivative of the function $\varphi(b)=\sqrt{b} \Gamma(b-1 / 2) / \Gamma(b)$ is

$$
\begin{aligned}
\frac{\varphi^{\prime}(b)}{\varphi(b)} & =\frac{1}{2 b}+\sum_{q \in \mathbb{N}}\left(\frac{1}{q+b}-\frac{1}{q+b-1 / 2}\right) \\
& \leq \frac{1}{2 b}+\int_{0}^{\infty}\left(\frac{1}{t+b}-\frac{1}{t+b-1 / 2}\right) d t \leq 0 .
\end{aligned}
$$

So for $b \geq 1$, we have

$$
\int_{0}^{\infty} \frac{d \tau}{\left(a^{2}+\tau^{2}\right)^{b}}=a^{1-2 b} \frac{\sqrt{\pi}}{2} \frac{\varphi(b)}{\sqrt{b}} \leq \frac{\sqrt{\pi}}{2} \frac{a^{1-2 b}}{\sqrt{b}} \varphi(1)=\frac{\pi}{2} \frac{a^{1-2 b}}{\sqrt{b}} .
$$

5. Bessel functions. Our main reference for this section is the book of Watson [W]. We recall here some properties of Bessel functions and we give an integral representation of these functions which is not given in [W].

Notation. From now on, if $c$ is a real number, we shall write $\int_{(c)} f(s) d s$ for $\int_{c-i \infty}^{c+i \infty} f(s) d s$.

Let $\nu$ be a real number. We define the Bessel function $J_{\nu}$ as (formula (8), $\S 3.1$ of $[\mathrm{W}])$ :

$$
\forall x \in \mathbb{R}, \quad J_{\nu}(x)=\sum_{m=0}^{\infty} \frac{(-1)^{m}(x / 2)^{\nu+2 m}}{m ! \Gamma(\nu+m+1)} .
$$

We recall the asymptotic expansion of $J_{\nu}(x)$ as $x \rightarrow \infty$ (§7.21 of [W]):

$$
\begin{aligned}
J_{\nu}(x) \sim & \left(\frac{2}{\pi x}\right)^{1 / 2}\left(\cos \left(x-\frac{\nu \pi}{2}-\frac{\pi}{4}\right) \sum_{m=0}^{\infty} \frac{(-1)^{m}(\nu, 2 m)}{(2 x)^{2 m}}\right. \\
& \left.-\sin \left(x-\frac{\nu \pi}{2}-\frac{\pi}{4}\right) \sum_{m=0}^{\infty} \frac{(-1)^{m}(\nu, 2 m+1)}{(2 x)^{2 m+1}}\right)
\end{aligned}
$$

with $(\nu, m)=\Gamma(\nu+m+1 / 2) / m ! \Gamma(\nu-m+1 / 2)$.

From a classical Mellin transform result (formula (7), $\S 6.5$ of [W]), for $\nu \geq 0$ and $0<c \leq \Re(\nu+1)$, we have

$$
x^{-\nu} J_{\nu}(x)=\frac{1}{2 i \pi} \int_{c-i \infty}^{c+i \infty} \frac{2^{s-\nu-1} \Gamma(s / 2)}{\Gamma(\nu-s / 2+1)} x^{-s} d s .
$$

REMARK 5.1. We recover here the function $f_{a, \nu}$ defined and studied in Section 3.1.

We will extend this result to the case $\nu \leq 0$. 
Lemma 5.1. Let $a>0$ and $\nu \geq 0$. If $f_{a, \nu}$ is defined as in Section 3.1, then we have

$$
\lim _{N \rightarrow \infty} \int_{(-2 N+1)} f_{a, \nu}(s) x^{-s} d s=0,
$$

uniformly for $x$ in compact subsets of $] 0, \infty[$.

Proof. We define $K_{N}(x):=\int_{(-2 N+1)} f_{a, \nu}(s) x^{-s} d s$. For $N \geq|\nu|+1$ and $z=1-s / 2$, from Proposition 3.2 we have

$$
\begin{aligned}
K_{N}(x) & =\frac{1}{2} \int_{(N+1 / 2)}\left(\frac{2}{a}\right)^{2-2 z-\nu} \frac{\Gamma(1-z)}{\Gamma(\nu+z)} x^{2 z-2} d z \\
& =O\left(\frac{1}{x}\left(\frac{e a x}{2}\right)^{2 N} \int_{(N+1 / 2)} \frac{|d z|}{|z|^{N}|\nu+z|^{N+\nu}}\right) .
\end{aligned}
$$

Furthermore, if $u, v \geq 1$, then $\frac{1}{u v} \leq \frac{1}{u}+\frac{1}{v}$, so we get

$$
K_{N}(x)=O\left(\frac{1}{x}\left(\frac{e a x}{2}\right)^{2 N}\left(\int_{(N+1 / 2)} \frac{|d z|}{|z|^{N}}+\int_{(N+1 / 2)} \frac{|d z|}{|\nu+z|^{N+\nu}}\right)\right) .
$$

By Lemma 4.2, we have

$$
\int_{(N+1 / 2)} \frac{|d z|}{|z|^{N}}=\int_{-\infty}^{\infty} \frac{d t}{\left((N+1 / 2)^{2}+t^{2}\right)^{N / 2}} \ll \frac{(N+1 / 2)^{1-N}}{\sqrt{N}}
$$

and

$$
\begin{aligned}
\int_{(N+1 / 2)} \frac{|d z|}{|\nu+z|^{N+\nu}} & =\int_{-\infty}^{\infty} \frac{d t}{\left((N+\nu+1 / 2)^{2}+t^{2}\right)^{(N+\nu) / 2}} \\
& \ll \frac{(N+\nu+1 / 2)^{1-N-\nu}}{\sqrt{N+\nu}} .
\end{aligned}
$$

If $N \geq 2|\nu|+1$, we get

$$
K_{N}(x)=O\left(\frac{1}{x} \sqrt{N}\left(\frac{e a x}{2}\right)^{2 N}\left(N^{-N}+(N / 2)^{-N / 2}\right)\right),
$$

and the integral $K_{N}(x)$ clearly tends to 0 as $N \rightarrow \infty$, uniformly for $x$ in compact subsets of $] 0, \infty[$. by

Definition 5.1. Let $a>0$ and $\nu \in \mathbb{R}$. We define the function $h_{0}=h_{0}^{\nu, a}$

$$
\forall x>0, \quad h_{0}(x):=x^{-\nu} J_{\nu}(a x) .
$$

Proposition 5.1. If $\nu \in \mathbb{R}$ and

$$
T \geq 1, \quad c<\nu, \quad \Lambda>0, \quad \Lambda \geq c,
$$


then the integral $\int_{C(T, c, \Lambda)} f(s) x^{-s} d s$ is convergent and its value does not depend on $T, c$ and $\Lambda$ satisfying (11). Furthermore,

$$
h_{0}(x)=\frac{1}{2 i \pi} \int_{C(T, c, \Lambda)} f_{a, \nu}(s) x^{-s} d s .
$$

Proof. In this proof, we will write $f$ for $f_{a, \nu}$.

Since the poles of the function $f_{a, \nu}(\alpha s+\kappa)$ are $(-2 n-\kappa) / \alpha, n \in \mathbb{N}$, the first part of the proposition follows from (6) and Lemma 4.1.

We define

$$
g(x):=\frac{1}{2 i \pi} \int_{C(T, c, \Lambda)} f(s) x^{-s} d s .
$$

If $N$ is a positive integer such that $N>\frac{1}{2}(1-\nu)$, then

$$
g(x)=\frac{1}{2 i \pi} \int_{C(T,-2 N+1, \Lambda)} f(s) x^{-s} d s .
$$

We apply the residue theorem to the function $f(s) x^{-s}$ on the rectangle $R:=[-2 N+1-i T, \Lambda-i T, \Lambda+i T,-2 N+1+i T]$ to get

$$
g(x)=\frac{1}{2 i \pi} \int_{(-2 N+1)} f(s) x^{-s} d s+\sum_{k=0}^{N-1} p_{k} x^{2 k},
$$

where

$$
p_{k}=\operatorname{Res}\left(f(s) x^{-s},-2 k\right)=\left(\frac{2}{a}\right)^{-2 k-\nu} \frac{(-1)^{k}}{k ! \Gamma(\nu+k+1)} .
$$

From Lemma 5.1, the integral $K_{N}(x):=\int_{(-2 N+1)} f(s) x^{-s} d s$ tends to 0 as $N \rightarrow \infty$, uniformly for $x$ in compact subsets of ]0, $\infty$ [. Letting $N \rightarrow \infty$ in (12), we get $g(x)=\sum_{m \geq 0} p_{m} x^{2 m}=x^{-\nu} J_{\nu}(a x)$.

We shall use some primitive functions of $h_{0}$ in the case $\nu>0$. To define them, we use some properties of the cosine and sine functions.

Definition 5.2. Let $a>0$ and $\nu \in \mathbb{R}$. For $t \in \mathbb{R}$, we define

$$
C(t)=\cos (a t-\nu \pi / 2-\pi / 4), \quad S(t)=\sin (a t-\nu \pi / 2-\pi / 4)
$$

and for $x>0$ and $R>-1 / 2$,

$$
\mathcal{C}_{R}(x)=-\int_{x}^{\infty} \sqrt{\frac{2}{\pi}} C(t) \frac{d t}{(a t)^{R+1 / 2}}, \quad \mathcal{S}_{R}(x)=-\int_{x}^{\infty} \sqrt{\frac{2}{\pi}} S(t) \frac{d t}{(a t)^{R+1 / 2}} .
$$

Using induction and partial integration, we establish the following asymptotic expansions. For all $n \in \mathbb{N}$ and all $R>-1 / 2$, if $\gamma_{k}^{(R)}:=$ 
$\Gamma(R+k+1 / 2) / \Gamma(R+1 / 2)$ for $k \in[0, n]$, then

$$
\begin{aligned}
\mathcal{C}_{R}(x)= & \frac{1}{a} \sqrt{\frac{2}{\pi}}\left(S(x) \sum_{k=0}^{n}(-1)^{k} \gamma_{2 k}^{(R)}(a x)^{-R-2 k-1 / 2}\right. \\
& \left.+C(x) \sum_{k=0}^{n-1}(-1)^{k+1} \gamma_{2 k+1}^{(R)}(a x)^{-R-2 k-3 / 2}\right)+O\left(x^{-R-2 n-3 / 2}\right),
\end{aligned}
$$

and

$$
\begin{aligned}
\mathcal{S}_{R}(x)= & -\frac{1}{a} \sqrt{\frac{2}{\pi}}\left(C(x) \sum_{k=0}^{n}(-1)^{k} \gamma_{2 k}^{(R)}(a x)^{-R-2 k-1 / 2}\right. \\
& \left.+S(x) \sum_{k=0}^{n-1}(-1)^{k} \gamma_{2 k+1}^{(R)}(a x)^{-R-2 k-3 / 2}\right)+O\left(x^{-R-2 n-3 / 2}\right)
\end{aligned}
$$

as $x \rightarrow \infty$. The implicit constants only depend on $R$ and $n$.

We can now deduce from (13) and (14) the following property.

Proposition-Definition 5.1. For $\nu>0$, we define by induction some primitive of $h_{0}$ of order $n$ by setting

$$
h_{n+1}(x)=-\int_{x}^{\infty} h_{n}(t) d t
$$

These integrals are convergent and for all $k \in \mathbb{N}$ there exist complex sequences $\left(c_{l}^{(k)}\right)_{l}$ and $\left(s_{l}^{(k)}\right)_{l}$ such that for all $M \in \mathbb{N}$, as $x \rightarrow \infty$,

$$
h_{k}(x)=\sum_{l=0}^{M} c_{l}^{(k)} C(x) x^{-l-\nu-1 / 2}+\sum_{l=0}^{M} s_{l}^{(k)} S(x) x^{-l-\nu-1 / 2}+O\left(x^{-\nu-M-3 / 2}\right) .
$$

Proposition 5.2. Let $k \in \mathbb{N}$ and $\nu>0$. If $0<c<\nu$ and $x>0$, then

$$
h_{k}(x)=\frac{1}{2 i \pi} \int_{c-i \infty}^{c+i \infty}(-1)^{k} \frac{\Gamma(s)}{\Gamma(s+k)} f(s+k) x^{-s} d s .
$$

Proof. We first prove by induction on $k$ that for all $s \in \mathbb{C}$ such that $0<\sigma<\nu+1 / 2$,

$$
\mathcal{M} h_{k}(s)=(-1)^{k} \frac{\Gamma(s)}{\Gamma(s+k)} f(s+k) .
$$

Then we use the inverse Mellin transform.

6. Expansion in a sum of hypergeometric functions. Let $F \in S^{\#}$ and $E(x)$ be the associated error term as defined in (3). In order to estimate $E(x)$, we define a smooth version of it. To go back to the initial function, we shall use finite differences. 
Definition 6.1. For $\varrho \in \mathbb{N}$, we define

$$
A^{\varrho}(x)=\frac{1}{\varrho !} \sum_{n \leq x}^{\prime} a_{n}(x-n)^{\varrho},
$$

the prime indicating that the last term has to be multiplied by $1 / 2$ if $\varrho=0$ and $x=n$. We also define

$$
S_{\varrho}(x)=\frac{1}{2 i \pi} \int_{C} \frac{F(s)}{s(s+1) \cdots(s+\varrho)} x^{s+\varrho} d s,
$$

where $C$ is a curve enclosing all the singularities of the integrand, and

$$
E^{\varrho}(x)=A^{\varrho}(x)-S_{\varrho}(x) .
$$

REMARK 6.1. $S_{\varrho}(x)$ is the sum of the residues of the integrand at its poles $-\varrho,-\varrho+1, \ldots,-1,0$ and 1 , so we have

$$
S_{\varrho}(x)=x^{\varrho} \operatorname{Res}\left(\frac{F(s) x^{s}}{s(s+1) \cdots(s+\varrho)}, 1\right)+\frac{1}{\varrho !} \sum_{k=0}^{\varrho}(-1)^{k} F(-k)\left(\begin{array}{l}
\varrho \\
k
\end{array}\right) x^{\varrho-k} .
$$

The first step consists in finding an expansion of $E^{\varrho}(x)$ as a sum of special functions.

Proposition 6.1. Let $\varrho \in \mathbb{N}^{*}, \varrho>d / 2$. For all $x>0$,

$$
E^{\varrho}(x)=\epsilon Q^{2 \varrho+1} \sum_{n \geq 1} \frac{\bar{a}_{n}}{n^{1+\varrho}} I_{\varrho}\left(\frac{n x}{Q^{2}}\right)
$$

where for $\varrho \in \mathbb{Z}, \varrho \geq-1$, the function $I_{\varrho}$ is defined as

$$
I_{\varrho}(x)=\frac{1}{2 i \pi} \int_{C_{1}} \frac{\bar{\gamma}(s)}{\gamma(1-s)} \frac{x^{1+\varrho-s} \Gamma(1-s)}{\Gamma(\varrho+2-s)} d s
$$

and $C_{1}=C(T, c, \Lambda)$ with

$$
T>T_{0}:=\max \left(\frac{|\Im \xi|}{d}, \max _{j=1}^{r} \frac{\left|\Im \mu_{j}\right|}{\lambda_{j}}\right), \quad c<\frac{\varrho}{d}+\frac{1}{2}, \quad \Lambda>\varrho+1 .
$$

Proof. If $c>1$ and $\varrho \geq 1$, then by a standard formula, we have

$$
A^{\varrho}(x)=\frac{1}{2 i \pi} \int_{\Re s=c} F(s) \frac{x^{s+\varrho}}{s(s+1) \cdots(s+\varrho)} d s,
$$

so that, if $g(s)=\frac{F(s)}{s(s+1) \cdots(s+\varrho)}$, then

$$
E^{\varrho}(x)=\frac{1}{2 i \pi} \int_{c-i \infty}^{c+i \infty} g(s) x^{s+\varrho} d s-\frac{1}{2 i \pi} \int_{C} g(s) x^{s+\varrho} d s .
$$

In view of Lemma 3.4, if $\sigma<0$ and $|\tau| \geq 1$, then $g(s)=O\left((1+|\tau|)^{d(1 / 2-\sigma)-\varrho-1}\right)$, so that, if $1 / 2-\varrho / d<c^{\prime}<0$ (such a $c^{\prime}$ exists if $\left.\varrho>d / 2\right), \Lambda^{\prime}<-\varrho, T^{\prime}>0$ 
and $\left.C_{2}:=\right] c^{\prime}-i \infty ; c^{\prime}-i T^{\prime} ; \Lambda^{\prime}-i T^{\prime} ; \Lambda^{\prime}+i T^{\prime} ; c^{\prime}+i T^{\prime} ; c^{\prime}+i \infty[$, then the integral

$$
\frac{1}{2 i \pi} \int_{C_{2}} \frac{F(s)}{s(s+1) \cdots(s+\varrho)} x^{s+\varrho} d s
$$

is convergent and by using the residue theorem between the lines $\sigma=c$ and $C_{2}$, we get

$$
E^{\varrho}(x)=\frac{1}{2 i \pi} \int_{C_{2}} \frac{F(s)}{s(s+1) \cdots(s+\varrho)} x^{s+\varrho} d s .
$$

We change the variable $s$ to $1-z$ and apply the functional equation to obtain

$$
E^{\varrho}(x)=\frac{1}{2 i \pi} \int_{C_{1}} \frac{\epsilon Q^{2 z-1} \bar{F}(z)}{(1-z)(-z) \cdots(1-z+\varrho)} x^{1-z+\varrho} \frac{\bar{\gamma}(z)}{\gamma(1-z)} d z
$$

where $C_{1}=C(T, c, \Lambda)$ with

$$
T>0, \quad c<\frac{\varrho}{d}+\frac{1}{2}, \quad \Lambda>\varrho+1 .
$$

By Lemmas 3.2 and 4.1, for $T>T_{0}=\max \left(|\Im \xi| / d, \max _{j=1}^{r}\left|\Im \mu_{j}\right| / \lambda_{j}\right), 1<$ $c<\varrho / d+1 / 2$ and $\Lambda>\varrho+1$, we get

$$
E^{\varrho}(x)=\epsilon Q^{2 \varrho+1} \sum_{n=1}^{\infty} \frac{\bar{a}_{n}}{n^{1+\varrho}} \frac{1}{2 i \pi} \int_{C_{1}} \frac{\bar{\gamma}(z)}{\gamma(1-z)} \frac{\left(n x / Q^{2}\right)^{1+\varrho-z}}{(1-z)(2-z) \cdots(1+\varrho-z)} d z .
$$

7. Study of the hypergeometric function $I_{\varrho}$. We now study the function $I_{\varrho}$. Kaczorowski and Perelli have studied a very similar function in [KP1] in order to describe functions of degree $d=1 \mathrm{in} S^{\#}$. Our work is strongly influenced by theirs. This function is a particular case of the Fox hypergeometric functions. These last functions have been studied in $[\mathrm{Br}]$. We will study the particular function $I_{\varrho}$ rather than extract the information we need from the very long and dense article $[\mathrm{Br}]$. In order to establish the asymptotic expansion of $I_{\varrho}$, we show that this function is closely related to Bessel functions.

We shall use the notations defined in Section 3.1.

The function $I_{\varrho}$ is closely related to the Mellin inverse transform of $G_{\varrho}$. We are going to approximate $G_{\varrho}$ by functions whose inverse Mellin transforms are known.

Recall that we have proved that

$$
G_{\varrho}(s)=\sum_{k=0}^{m} F_{k}(s)+r_{m}(s),
$$


with

$$
F_{k}(s)=\frac{(-1)^{k} e_{k} f(\alpha s+\kappa)}{(\alpha s+\kappa-k) \cdots(\alpha s+\kappa-1)}, \quad r_{m}(s)=O\left(|\tau|^{d(\sigma-1 / 2)-\varrho-m-2}\right) .
$$

If $T>T_{0}, \Lambda>\max (\varrho+1,(m-\Re \kappa) / d), c<1 / 2+\varrho / d, c$ is not a pole of $r_{k}$ and $C_{1}=C(T, c, \Lambda)$ is the path as in Definition 4.1, then the integrals

$$
H_{k}(x):=\frac{1}{2 i \pi} \int_{C_{1}} F_{k}(s) x^{1+\varrho-s} d s \quad \text { and } \quad R_{k}(x):=\int_{C_{1}} r_{k}(s) x^{1+\varrho-s} d s
$$

are convergent. With these notations, for $x>0$ and $m \in \mathbb{N}$ we have

$$
I_{\varrho}(x)=\sum_{k=0}^{m} H_{k}(x)+R_{m}(x) \text {. }
$$

To estimate $R_{m}$, we move the path of integration to the right so that the integral remains convergent. By Lemma 4.1 , for $m \in \mathbb{N}$ and $\varepsilon>0$ we get

$$
R_{m}(x)=O_{\varepsilon}\left(x^{1 / 2+\varrho-(\varrho+m+1) / d+\varepsilon}\right) \quad \text { as } x \rightarrow \infty .
$$

Proposition 7.1. If $T \geq 1, \Lambda>\max (0, c)$ and $c<\nu$, then

$$
H_{k}(x)=\frac{e_{k}}{d 2 i \pi} x^{1+\varrho+(\kappa-k) / d} \int_{C(T, c, \Lambda)} \frac{(-1)^{k} f(z+k)}{z(z+1) \cdots(z+k-1)}\left(x^{1 / d}\right)^{-z} d z .
$$

Proof. By (6),

$$
F_{k}(s) \ll|\tau|^{d(\sigma-1 / 2)-\varrho-k-1} \quad \text { as }|\tau| \rightarrow \infty,
$$

so if $T>T_{0}:=\max \left(|\Im \xi| / d, \max _{j=1}^{r}\left|\Im \mu_{j}\right| / \lambda_{j}\right), c<(1 / d)(\varrho+k)+1 / 2$ and $\Lambda>\max (\varrho+1,(k-\Re \kappa) / d)$, by Lemma 4.1 , we have

$$
\begin{aligned}
H_{k}(x) & =\frac{1}{2 i \pi} \int_{C(T, c, \Lambda)} F_{k}(s) x^{1+\varrho-s} d s \\
& =\frac{1}{2 i \pi} \int_{C(T, c, \Lambda)} e_{k} f(\alpha s+\kappa) \frac{(-1)^{k} x^{1+\varrho-s}}{(\alpha s+\kappa-k) \cdots(\alpha s+\kappa-1)} d s .
\end{aligned}
$$

We change the variable $s$ to $z=\alpha s+\kappa-k$ to obtain

$$
H_{k}(x)=\frac{e_{k} / d}{2 i \pi} x^{1+\varrho+(\kappa-k) / d} \int_{C\left(T^{\prime}, c^{\prime}, \Lambda^{\prime}\right)} \frac{(-1)^{k} f(z+k)}{z(z+1) \cdots(z+k-1)}\left(x^{1 / d}\right)^{-z} d z
$$

for any real $T^{\prime}, c^{\prime}$ and $\Lambda^{\prime}$ satisfying $T^{\prime} \geq 1, \Lambda^{\prime}>\max \left(0, c^{\prime}\right)$ and $c^{\prime}<\nu$.

We now establish a link between $H_{k}$ and $h_{k}$ in case $\nu>0$, i.e. in case $\varrho>-\Re \kappa-d / 2$.

Proposition 7.2. If $\nu>0$, then for $x>0$ we have

$$
H_{k}(x)=\frac{e_{k}}{d} x^{1+\varrho+(\kappa-k) / d} h_{k}\left(x^{1 / d}\right) \text {. }
$$


Proof. By Proposition 7.1, we have

$$
H_{k}(x)=\frac{e_{k} / d}{2 i \pi} x^{1+\varrho+(\kappa-k) / d} \int_{C(T, c, \Lambda)} \frac{(-1)^{k} f(z+k)}{z(z+1) \cdots(z+k-1)}\left(x^{1 / d}\right)^{-z} d z .
$$

By Lemma 4.1, since the real parts of the poles of the integrand are less than 0 , and since we have

$$
\left|\frac{(-1)^{k} f(z+k)}{z(z+1) \cdots(z+k-1)}\right| \asymp|\tau|^{\sigma-\nu-1},
$$

we can choose $0<c=\Lambda<\nu$ because $\nu>0$, and so

$$
\begin{aligned}
H_{k}(x) & =\frac{e_{k} / d}{2 i \pi} x^{1+\varrho+(\kappa-k) / d} \int_{c-i \infty}^{c+i \infty} \frac{(-1)^{k} f(z+k)}{z(z+1) \cdots(z+k-1)}\left(x^{1 / d}\right)^{-z} d z \\
& =\delta e_{k} x^{1+\varrho+\delta(\kappa-k)} h_{k}\left(x^{1 / d}\right) .
\end{aligned}
$$

For $k=0$ and $\nu \in \mathbb{R}$, we also have

$$
H_{0}(x)=\frac{e_{0}}{d} x^{1+\varrho+\kappa / d} h_{0}\left(x^{1 / d}\right) .
$$

We shall now deduce from the link between $I_{\varrho}$ and $h_{k}$ an asymptotic expansion of $I_{\varrho}$ in case $\nu>0$ and an asymptotic equivalent of $I_{0}$ in case $\nu \leq 0$.

Notation. We write

$$
\delta=1 / d, \quad \omega=1+\varrho+(\kappa-\nu-1 / 2) \delta=(\varrho+1 / 2)(1-\delta)-i \delta \Im \xi .
$$

Theorem 7.1. Assume $\varrho>-1-\Re \xi-d / 2$ (i.e. $\nu>0$ ). There exist complex sequences $\left(\delta_{n}\right)_{n}$ and $\left(\delta_{n}^{\prime}\right)_{n}$ such that for all $m \in \mathbb{N}$ and all $\varepsilon>0$, as $x \rightarrow \infty$,

$$
I_{\varrho}(x)=e^{i a x^{\delta}} \sum_{n=0}^{m} \delta_{n} x^{\omega-n \delta}+e^{-i a x^{\delta}} \sum_{n=0}^{m} \delta_{n}^{\prime} x^{\omega-n \delta}+O\left(x^{\Re \omega-(m+1 / 2) \delta+\varepsilon}\right) .
$$

Proof. By Proposition 7.2, (17) and (16), we have

$$
\begin{aligned}
I_{\varrho}(x) & =\sum_{k=0}^{m} \delta e_{k} x^{1+\varrho+(\kappa-k) \delta} h_{k}\left(x^{\delta}\right)+O\left(x^{1 / 2+\varrho-(\varrho+m+1) \delta+\varepsilon}\right) \\
& =\sum_{k=0}^{m} \delta e_{k} x^{1+\varrho+(\kappa-k) \delta} h_{k}\left(x^{\delta}\right)+O\left(x^{\Re \omega-\delta(m+1 / 2)+\varepsilon}\right) .
\end{aligned}
$$

Using the asymptotic expansion of $h_{k}$ (see Proposition-Definition 5.1) we show that there exist complex sequences $\left(\delta_{n}\right)_{n}$ and $\left(\delta_{n}^{\prime}\right)_{n}$ such that for 
all $m \in \mathbb{N}$

$$
\begin{aligned}
I_{\varrho}(x)= & C\left(x^{\delta}\right) \sum_{n=0}^{m} \delta_{n} x^{1+\varrho+(\kappa-\nu-n-1 / 2) \delta}+S\left(x^{\delta}\right) \sum_{n=0}^{m} \delta_{n}^{\prime} x^{1+\varrho+(\kappa-\nu-n-1 / 2) \delta} \\
& +O\left(x^{1 / 2+\varrho-(\varrho+m+1) \delta+\varepsilon}\right)
\end{aligned}
$$

so there exist complex sequences $\left(\eta_{n}\right)_{n}$ and $\left(\eta_{n}^{\prime}\right)_{n}$ such that for all $m \in \mathbb{N}$,

$$
I_{\varrho}(x)=e^{i a x^{\delta}} \sum_{n=0}^{m} \delta_{n} x^{\omega-n \delta}+e^{-i a x^{\delta}} \sum_{n=0}^{m} \delta_{n}^{\prime} x^{\omega-n \delta}+O\left(x^{\Re \omega-(m+1 / 2) \delta+\varepsilon}\right) .
$$

Proposition 7.3. Let $\nu \in \mathbb{R}$. Then

$$
I_{0}(x)=O\left(x^{(1-\delta) / 2}\right) \quad \text { as } x \rightarrow \infty .
$$

Proof. By (16)-(18), for all $\varepsilon>0$ we have

$$
I_{0}(x)=\delta e_{0} x^{1+\delta \kappa} h_{0}\left(x^{\delta}\right)+O_{\varepsilon}\left(x^{1 / 2-\delta+\varepsilon}\right) .
$$

But $h_{0}(x)=O\left(x^{-\nu-1 / 2}\right)=O\left(x^{-\Re \kappa-d / 2-1 / 2}\right)$, so $I_{0}(x)=O\left(x^{(1-\delta) / 2}\right)+$ $O_{\varepsilon}\left(x^{1 / 2-\delta+\varepsilon}\right)$. We choose $0<\varepsilon<\delta / 2$ to get the final result.

Proposition 7.4. If $\varrho \in \mathbb{N}$, then the function $I_{\varrho}$ has a $\varrho$ th derivative and for all $k \in \mathbb{N}, k \leq \varrho$, we have

$$
I_{\varrho}^{(k)}(y)=I_{\varrho-k}(y) .
$$

Proof. Assume that $\varrho \in \mathbb{N}$ and $T, c$ and $\Lambda$ satisfy (15). Then

$$
I_{\varrho}(x)=\frac{1}{2 i \pi} \int_{C(T, c, \Lambda)} G_{\varrho}(s) x^{1+\varrho-s} d s .
$$

We have

$$
\frac{d}{d x}\left(G_{\varrho}(s) x^{1+\varrho-s}\right)=(1+\varrho-s) G_{\varrho}(s) x^{\varrho-s}
$$

and the integral

$$
\int_{C(T, c, \Lambda)}(1+\varrho-s) G_{\varrho}(s) x^{\varrho-s} d s
$$

is convergent uniformly for $x$ in compact subsets of $[0, \infty[$. So we have

$$
I_{\varrho}^{\prime}(x)=\frac{1}{2 i \pi} \int_{C(T, c, \Lambda)}(1+\varrho-s) G_{\varrho}(s) x^{\varrho-s} d s .
$$

$\operatorname{But}(1+\varrho-s) G_{\varrho}(s)=G_{\varrho-1}(s)$ so

$$
I_{\varrho}^{\prime}(x)=\frac{1}{2 i \pi} \int_{C(T, c, \Lambda)} G_{\varrho-1}(s) x^{\varrho-s} d s .
$$

Since $T, c$ and $\Lambda$ satisfy conditions (15) with $\varrho-1$ instead of $\varrho$, we have

$$
I_{\varrho}^{\prime}(x)=I_{\varrho-1}(x) \text {. }
$$

An iteration gives the conclusion. 


\section{Estimation of the error term}

8.1. Estimation of the error term with Landau's method. In this section we shall prove Theorem 2.1. Let $F \in S^{\#}$ be of degree $d \geq 2$. Using arguments similar to the one used in the proof of the Perron formula (see [T, Section I.2, Theorem 1]), we prove that for all $\varepsilon>0, x \geq 1$ and $T \geq 1$,

$$
\begin{aligned}
\sum_{n \leq x}^{\prime} a_{n}= & \frac{1}{2 i \pi} \int_{1+\varepsilon-i T}^{1+\varepsilon+i T} \frac{x^{s} F(s)}{s} d s+O\left(x^{1+\varepsilon} \sum_{\substack{n=1 \\
n \neq x}}^{\infty} \frac{\left|a_{n}\right|}{n^{1+\varepsilon}(T|\log (x / n)|)}\right) \\
& +O\left(\frac{\left|a_{x}\right|}{T}\right) \\
= & \frac{1}{2 i \pi} \int_{1+\varepsilon-i T}^{1+\varepsilon+i T} \frac{x^{s} F(s)}{s} d s+O\left(\frac{x^{1+\varepsilon}}{T}\right)+O\left(\frac{\left|a_{x}\right|}{T}\right),
\end{aligned}
$$

where $a_{x}=0$ if $x \notin \mathbb{N}$. Applying the residue theorem, we get

$$
\begin{aligned}
\frac{1}{2 i \pi} \int_{1+\varepsilon-i T}^{1+\varepsilon+i T} \frac{x^{s} F(s)}{s} d s= & S(x)+\frac{1}{2 i \pi} \int_{1+\varepsilon-i T}^{-\varepsilon-i T} \frac{x^{s} F(s)}{s} d s \\
& +\frac{1}{2 i \pi} \int_{-\varepsilon-i T}^{-\varepsilon+i T} \frac{x^{s} F(s)}{s} d s+\frac{1}{2 i \pi} \int_{-\varepsilon+i T}^{1+\varepsilon+i T} \frac{x^{s} F(s)}{s} d s .
\end{aligned}
$$

Let us estimate the horizontal integrals. If $s=\sigma+i T$ with $T \geq 1$ and $-\varepsilon \leq \sigma \leq 1+\varepsilon$, by Lemma 3.4 we have

$$
\frac{x^{s}}{s} F(s)=O\left(T^{d(1+\varepsilon) / 2-1}\left(x T^{-d / 2}\right)^{\sigma}\right),
$$

and therefore,

$$
\begin{aligned}
\int_{-\varepsilon \pm i T}^{1+\varepsilon \pm i T} \frac{x^{s} F(s)}{s} d s & =O\left(T^{d(1+\varepsilon) / 2-1}\left(\frac{x^{1+\varepsilon}}{T^{d(1+\varepsilon) / 2}}+\frac{x^{-\varepsilon}}{T^{-d \varepsilon / 2}}\right)\right) \\
& =O\left(\frac{1}{T} x^{1+\varepsilon}\right)+O\left(x^{-\varepsilon} T^{d(1+2 \varepsilon) / 2-1}\right) .
\end{aligned}
$$

Now, let us estimate the vertical integral. Using the functional equation and Lemma 3.2, we have

$$
\begin{aligned}
F(s)= & \epsilon Q^{2 s-1} \frac{\bar{\gamma}(1-s)}{\gamma(s)} \bar{F}(1-s) \\
= & \epsilon Q^{2 s-1} c(\sigma, \tau)|\tau|^{d(1 / 2-\sigma)} e^{i(-d \tau \log |\tau|+(\log \beta+d) \tau-\Im \xi \log |\tau|)} \\
& \times \bar{F}(1-s)(1+O(1 /|\tau|)) .
\end{aligned}
$$


So, for $s=-\varepsilon+i \tau$, we have

$$
\begin{aligned}
& F(s) \frac{x^{s}}{s} \\
& \quad=A x^{-\varepsilon}|\tau|^{d(1 / 2+\varepsilon)-1} \sum_{n \geq 1} \frac{\bar{a}_{n}}{n^{1+\varepsilon}} e^{i\left(-(d \tau+\Im \xi) \log |\tau|+\left(\log \frac{\beta Q^{2} x}{n}+d\right) \tau\right)}(1+O(1 /|\tau|)),
\end{aligned}
$$

where $A$ is a constant depending on $\varepsilon$ and the sign of $\tau$. We have

$$
\begin{aligned}
& \int_{-\varepsilon-i T}^{-\varepsilon+i T} F(s) \frac{x^{s}}{s} d s \\
& =A_{1} x^{-\varepsilon} \sum_{n \geq 1} \frac{\bar{a}_{n}}{n^{1+\varepsilon}} \int_{1 / d}^{T} \tau^{d(1 / 2+\varepsilon)-1} e^{i\left(-(d \tau+\Im \xi) \log \tau+\left(\log \frac{\beta Q^{2} x}{n}+d\right) \tau\right)} d \tau \\
& \quad+A_{2} x^{-\varepsilon} \sum_{n \geq 1} \frac{\bar{a}_{n}}{n^{1+\varepsilon}} \int_{1 / d}^{T} \tau^{d(1 / 2+\varepsilon)-1} e^{i\left((d \tau-\Im \xi) \log \tau-\left(\log \frac{\beta Q^{2} x}{n}+d\right) \tau\right)} d \tau \\
& \quad+O\left(x^{-\varepsilon} \sum_{n \geq 1} \int_{1 / d}^{T} \tau^{d(1 / 2+\varepsilon)-2} d \tau\right)+O\left(x^{-\varepsilon}\right)
\end{aligned}
$$

With $u=d \tau$, we have

$$
\begin{aligned}
& \int_{-\varepsilon-i T}^{-\varepsilon+i T} F(s) \frac{x^{s}}{s} d s \\
& =B_{1} x^{-\varepsilon} \sum_{n \geq 1} \frac{\bar{a}_{n}}{n^{1+\varepsilon}} \int_{1}^{d T} u^{d(1 / 2+\varepsilon)-1} e^{i\left(-(u+\Im \xi) \log u+\left(\log d+\log \frac{\beta Q^{2} x}{n} / d+1\right) u\right)} d u \\
& \quad+B_{2} x^{-\varepsilon} \sum_{n \geq 1} \frac{\bar{a}_{n}}{n^{1+\varepsilon}} \int_{1}^{d T} u^{d(1 / 2+\varepsilon)-1} e^{i\left((u-\Im \xi) \log u-\left(\log \frac{\beta Q^{2} x}{n} / d+1+\log d\right) u\right)} d u \\
& \quad+O\left(x^{-\varepsilon} T^{d(1 / 2+\varepsilon)-1}\right)+O\left(x^{-\varepsilon}\right) \\
& =B_{1} x^{-\varepsilon} \sum_{n \geq 1} \frac{\bar{a}_{n}}{n^{1+\varepsilon}} \int_{1}^{d T} u^{d(1 / 2+\varepsilon)-1} e^{i(\varphi(u)-\Im \xi \log u)} d u \\
& \quad+B_{2} x^{-\varepsilon} \sum_{n \geq 1} \frac{\bar{a}_{n}}{n^{1+\varepsilon}} \int_{1}^{d T} u^{d(1 / 2+\varepsilon)-1} e^{i(-\varphi(u)-\Im \xi \log u)} d u \\
& \quad+O\left(x^{-\varepsilon} T^{d(1 / 2+\varepsilon)-1}\right)+O\left(x^{-\varepsilon}\right)
\end{aligned}
$$

with $\varphi(u)=-u \log u+\left(\log \frac{\beta Q^{2} x}{n} / d+1+\log d\right) u$. 
According to Hilfsatz 10 of [Lan], for $U \geq 1, \delta \geq 0$ and $w \in \mathbb{R}$ we have

$$
\left|\int_{1}^{U} u^{\delta} e^{ \pm i u(\log u-w)} d u\right|<23 U^{1 / 2+\delta}
$$

Applying this lemma with $\delta=d(1 / 2+\varepsilon)-1$ and $w=\log \frac{\beta Q^{2} x}{n} / d+1+\log d$, for $U \geq 1$ and $d \geq 2$ we get

$$
\int_{1}^{U} u^{d(1 / 2+\varepsilon)-1} e^{ \pm i \varphi(u)} d u<23 U^{d(1 / 2+\varepsilon)-1 / 2} .
$$

Therefore,

$$
\int_{1}^{d T} u^{d(1 / 2+\varepsilon)-1} e^{i( \pm \varphi(u)-\Im \xi \log u)} d u=O\left(T^{d(1 / 2+\varepsilon)-1 / 2}\right)
$$

and so

$$
\int_{-\varepsilon-i T}^{-\varepsilon+i T} F(s) \frac{x^{s}}{s} d s=O\left(x^{-\varepsilon} T^{d(1 / 2+\varepsilon)-1 / 2}\right) .
$$

Finally, we have proved that if $d \geq 2$, then

$$
\sum_{n \leq x}^{\prime} a_{n}=S(x)+O\left(\frac{1}{T} x^{1+\varepsilon}\right)+O\left(x^{-\varepsilon} T^{(d-1) / 2+\varepsilon}\right)+O\left(\frac{\left|a_{x}\right|}{T}\right)
$$

so with $T=x^{(d+1) / 2}$, since $a_{n}=o\left(n^{1+\varepsilon}\right)$, we have

$$
\sum_{n \leq x}^{\prime} a_{n}=S(x)+O\left(x^{(d-1) /(d+1)+\varepsilon}\right) .
$$

REMARK 8.1. With a similar method combined with the van der Corput method used instead of Hilfsatz 10, we could prove that for $1 \leq d<2$, we have the estimate $E(x)=O\left(x^{d / 4+\varepsilon}\right)$.

8.2. Method used by Chandrasekharan and Narasimhan. We shall use finite differences to establish a link between $E(x)$ and its smooth version $E^{\varrho}(x)$.

If $\varrho$ is an integer, $\lambda>0$ and $0<\varrho \lambda<x$, the $\varrho$ th finite difference of the real function $f$ is defined as

$$
\Delta_{\lambda}^{\varrho} f(x)=\sum_{\nu=0}^{\varrho}(-1)^{\varrho-\nu}\left(\begin{array}{l}
\varrho \\
\nu
\end{array}\right) f(x+\lambda \nu) .
$$

By formula (8) of [CN2], we have

$$
\begin{aligned}
E(x)=E^{0}(x)= & \lambda^{-\varrho} \Delta_{\lambda}^{\varrho}\left(A^{\varrho}(x)-S_{\varrho}(x)\right) \\
& +O\left(\lambda \log ^{m_{F}-1} x\right)+O\left(\sum_{x<n \leq x+\varrho \lambda}\left|a_{n}\right|\right) .
\end{aligned}
$$


Notation. In case $d>1$, we define $\lambda(x)=x^{1-\delta-\eta}$, where $\delta=1 / d$ and $\eta>0$, and choose a convenient $\eta$ for each case.

DeFinition 8.1. We define

$$
W(x)=\Delta_{\lambda}^{\varrho}\left(A^{\varrho}(x)-S_{\varrho}(x)\right), \quad V(x)=E(x)-\lambda^{-\varrho} W(x) .
$$

We assume $d>1$. In order to estimate $W(y)$, we shall use Proposition 6.1, the asymptotic expansion of $I_{\varrho}$ and some properties of finite differences. We follow the method used by Chandrasekharan and Narasimhan in $[\mathrm{CN} 1]$.

Notation. For all natural $n$, we write $\alpha_{n}=n / Q^{2}$.

Since $W(y)=\Delta_{\lambda}^{\varrho}\left(A^{\varrho}(y)-S_{\varrho}(y)\right)$, by Proposition 6.1, for $\varrho>d / 2$ and $y>0$ we have

$$
W(y)=\alpha Q^{-1} \sum_{n=1}^{\infty} \frac{\bar{a}_{n}}{\alpha_{n}^{1+\varrho}} \Delta_{\lambda}^{\varrho} I_{\varrho}\left(\alpha_{n} y\right) .
$$

We now estimate $\Delta_{\lambda}^{\varrho} I_{\varrho}(y)$. Assume that $\varrho \in \mathbb{N}, \lambda>0, y \in \mathbb{R}$ and $f$ has a $\varrho$ th derivative on $] 0, \infty[$. Then

$$
\Delta_{\lambda}^{\varrho} f(y)=\int_{y}^{y+\lambda} \int_{t_{1}}^{t_{1}+\lambda} \cdots \int_{t_{\varrho-1}}^{t_{\varrho-1}+\lambda} f^{(\varrho)}\left(t_{\varrho}\right) d t_{\varrho} \cdots d t_{2} d t_{1} .
$$

In particular,

$$
\left|\Delta_{\lambda}^{\varrho} f(y)\right| \leq \lambda^{\varrho} \sup _{y \leq t \leq y+\varrho \lambda}\left|f^{(\varrho)}(t)\right| .
$$

Applying this to $I_{\varrho}$, by Proposition 7.4 we get

$$
\left|\Delta_{\lambda}^{\varrho} I_{\varrho}(y)\right| \leq \lambda^{\varrho} \sup _{y \leq t \leq y+\varrho \lambda}\left|I_{0}(t)\right| .
$$

By Proposition 7.3, $I_{0}(x)=O\left(x^{(1-\delta) / 2}\right)$ so if $y \geq \lambda \varrho$, we have

$$
\left|\Delta_{\lambda}^{\varrho} I_{\varrho}(y)\right| \leq \lambda^{\varrho} O\left(y^{(1-\delta) / 2}\right) .
$$

On the other hand, by Theorem 7.1 we have

$$
\left|\Delta_{\lambda}^{\varrho} I_{\varrho}(y)\right|=O\left(\left|I_{\varrho}(y)\right|\right)=O\left(y^{\Re \omega}\right)=O\left(y^{(\varrho+1 / 2)(1-\delta)}\right) .
$$

For $\varrho>d / 2, z>0$ and $y>0$, we have

$$
\begin{aligned}
W(y) & =\alpha Q^{-1} \sum_{n=1}^{\infty} \frac{\bar{a}_{n}}{\alpha_{n}^{1+\varrho}} \Delta_{\lambda}^{\varrho} I_{\varrho}\left(\alpha_{n} y\right) \\
& =\alpha Q^{-1} \sum_{\alpha_{n} \leq z} \frac{\bar{a}_{n}}{\alpha_{n}^{1+\varrho}} \Delta_{\lambda}^{\varrho} I_{\varrho}\left(\alpha_{n} y\right)+\alpha Q^{-1} \sum_{\alpha_{n}>z} \frac{\bar{a}_{n}}{\alpha_{n}^{1+\varrho}} \Delta_{\lambda}^{\varrho} I_{\varrho}\left(\alpha_{n} y\right) .
\end{aligned}
$$


Using (20) in the first sum and (21) in the second one, we get

$$
\begin{aligned}
W(y) & =O\left(\sum_{\alpha_{n} \leq z} \frac{\left|a_{n}\right|}{\alpha_{n}^{1+\varrho}} \alpha_{n}^{\varrho} \lambda^{\varrho}\left(\alpha_{n} y\right)^{(1-\delta) / 2}\right)+O\left(\sum_{\alpha_{n}>z} \frac{\left|a_{n}\right|}{\alpha_{n}^{1+\varrho}}\left(\alpha_{n} y\right)^{(\varrho+1 / 2)(1-\delta)}\right) \\
& =O\left(\sum_{\alpha_{n} \leq z} \frac{\left|a_{n}\right|}{\alpha_{n}^{(1+\delta) / 2}} \lambda^{\varrho} y^{(1-\delta) / 2}\right)+O\left(\sum_{\alpha_{n}>z} \frac{\left|a_{n}\right|}{\alpha_{n}^{\varrho \delta+(1-\delta) / 2}} y^{(\varrho+1 / 2)(1-\delta)}\right) .
\end{aligned}
$$

If $\varrho>(d+1) / 2$, using the definition $\lambda=y^{1-\delta-\eta}$ and choosing $z=y^{d \eta}$, since $\sum_{n=1}^{\infty}\left|a_{n}\right| n^{-1-\varepsilon}<\infty$ for all $\varepsilon>0$, we get

$$
W(y)=O\left(z^{1+\varepsilon-(1+\delta) / 2} y^{(\varrho+1 / 2)(1-\delta)-\varrho \eta}\right)+O\left(z^{1+\varepsilon-\varrho \delta-\delta / 2} y^{(\varrho+1 / 2)(1-\delta)}\right)
$$

for all $\varepsilon>0$ and so

$$
W(y)=O\left(y^{(\varrho+1 / 2)(1-\delta-\eta)+d \eta / 2+\varepsilon}\right) .
$$

We can now prove the result announced in Remark 2.1: for any function $F \in S^{\#}$ such that $\sum_{n \leq x}\left|a_{n}\right|^{2} \ll n^{1+\varepsilon}$ for all $\varepsilon>0$ (also for functions of degree $d<2$ ), for any $x \geq 1$ and any $\varepsilon>0$, we have

$$
E(x)=O\left(x^{(d-1) /(d+1)+\varepsilon}\right) .
$$

Let $F$ be such a function. By Cauchy's inequality, we have

$$
\left(\sum_{y<n \leq y+\varrho \lambda}\left|a_{n}\right|\right)^{2} \leq \varrho \lambda\left(\sum_{y<n \leq y+\varrho \lambda}\left|a_{n}\right|^{2}\right) \ll y^{2-\delta-\eta+\varepsilon}
$$

for all $\varepsilon>0$, so

$$
V(y) \ll y^{1-(\delta+\eta) / 2+\varepsilon} .
$$

Since $E(x)=V(x)+\lambda^{-\varrho} W(x)$, by (25) and (23) we have, for all $\varepsilon>0$,

$$
E(x)=O\left(x^{(1-\delta-\eta) / 2+d \eta / 2+\varepsilon}\right)+O\left(x^{1-(\delta+\eta) / 2+\varepsilon}\right) .
$$

We choose $\eta=\delta$ to get $E(x)=O\left(x^{1-\delta+\varepsilon}\right)$.

If we assume that $a_{n}=O\left(n^{\varepsilon}\right)$ for all $\varepsilon>0$, then $V(x) \ll x^{1-\delta-\eta+\varepsilon}$, so by (23), for all $\varepsilon>0$,

$$
E(x)=O\left(x^{(1-\delta-\eta) / 2+d \eta / 2+\varepsilon}\right)+O\left(x^{1-(\delta+\eta) / 2+\varepsilon}\right) .
$$

We choose $\eta=(1-\delta) /(d+1)$ to get $E(x)=O\left(x^{(d-1) /(d+1)+\varepsilon}\right)$.

9. Estimation of the mean square of the error term. We shall now estimate the integral $\int_{0}^{x}|E(y)|^{2} d y$ and prove Theorem 2.2. Since the estimate of Theorem 2.2 is obvious for functions of degree $d \leq 1$ in $S^{\#}$, we shall assume that $F \in S^{\#}$ is of degree $d>1$ and $\sum_{n \leq x}\left|a_{n}\right|^{2} \ll x^{1+\varepsilon}$ for all $\varepsilon>0$. We have

$$
\int_{1}^{x}|E(y)|^{2} d y \leq 4 \max \left(\int_{1}^{x} \lambda^{-2 \varrho}|W(y)|^{2} d y, \int_{1}^{x}|V(y)|^{2} d y\right) .
$$


9.1. Estimation of the second integral. As $x \rightarrow \infty$, we have

$$
\begin{aligned}
\int_{2}^{x} \lambda(y)^{2} \log ^{2 m_{F}-2} y d y & =\int_{2}^{x} y^{2(1-\delta-\eta)} \log ^{2 m_{F}-2} y d y \\
& \ll x^{3-2 \delta-2 \eta} \log ^{2 m_{F}-2} x+1 .
\end{aligned}
$$

By (24), we have

$$
\begin{aligned}
\int_{1}^{x}\left(\sum_{y<n \leq y+\varrho \lambda}\left|a_{n}\right|\right)^{2} d y & =O\left(\int_{1}^{x} y^{1-\delta-\eta}\left(\sum_{y<n \leq y+\varrho \lambda}\left|a_{n}\right|^{2}\right) d y\right) \\
& =O\left(\sum_{1<n \leq x+\varrho x^{1-\delta-\eta}}\left|a_{n}\right|^{2} \int_{n-\varrho n^{1-\delta-\eta}}^{n} y^{1-\delta-\eta} d y\right) \\
& =O\left(\sum_{1<n \leq x+\varrho x^{1-\delta-\eta}}\left|a_{n}\right|^{2} n^{2(1-\delta-\eta)}\right) .
\end{aligned}
$$

So, for all $\varepsilon>0$, as $x \rightarrow \infty$,

$$
\begin{aligned}
\int_{1}^{x}\left(\sum_{y<n \leq y+\varrho \lambda}\left|a_{n}\right|\right)^{2} d y & =O\left(\left(x+\varrho x^{1-\delta-\eta}\right)^{2(1-\delta-\eta)+1+\varepsilon}\right) \\
& =O\left(x^{3-2 \delta-2 \eta+\varepsilon}\right) .
\end{aligned}
$$

Finally, by (19), for all $\varepsilon>0$, we have

$$
\int_{1}^{x}|V(y)|^{2} d y=O\left(x^{3-2 \delta-2 \eta+\varepsilon}+1\right) .
$$

9.2. Estimation of the first integral. To estimate $\int_{1}^{x} \lambda^{-2 \varrho}|W(y)|^{2} d y$, we shall use Proposition 6.1, the asymptotic expansion of $I_{\varrho}$ and some properties of finite differences. We follow the method used by Chandrasekharan and Narasimhan in [CN2].

By $(22)$, for $\varrho>d / 2$ and $y>0$, we have

$$
|W(y)|^{2}=Q^{2} \sum_{m=1}^{\infty} \sum_{n=1}^{\infty} \frac{\bar{a}_{m} a_{n}}{\left(\alpha_{m} \alpha_{n}\right)^{1+\varrho}} \Delta_{\lambda}^{\varrho} I_{\varrho}\left(\alpha_{m} y\right) \Delta_{\lambda}^{\varrho} \bar{I}_{\varrho}\left(\alpha_{n} y\right) .
$$

DeFinition 9.1. We write

$$
\begin{aligned}
& W_{1}(y)=\sum_{n=1}^{\infty} \frac{\left|a_{n}\right|^{2}}{\alpha_{n}^{2(1+\varrho)}}\left|\Delta_{\lambda}^{\varrho} I_{\varrho}\left(\alpha_{n} y\right)\right|^{2}, \\
& W_{2}(y)=\sum_{n \neq m} \frac{\bar{a}_{m} a_{n}}{\left(\alpha_{m} \alpha_{n}\right)^{1+\varrho}} \Delta_{\lambda}^{\varrho} I_{\varrho}\left(\alpha_{m} y\right) \Delta_{\lambda}^{\varrho} \bar{I}_{\varrho}\left(\alpha_{n} y\right) .
\end{aligned}
$$

We shall estimate $\int_{1}^{x} \lambda^{-2 \varrho} W_{1}(y) d y$ and $\int_{1}^{x} \lambda^{-2 \varrho} W_{2}(y) d y$ separately. 


\subsubsection{Estimation of diagonal terms}

Proposition 9.1. If $\varrho \in \mathbb{N}$ and $x>1$, then

$$
\int_{1}^{x} \lambda^{-2 \varrho} W_{1}(y) d y=O\left(x^{2-\delta}\right) .
$$

Proof. By (20), we have

$$
\begin{aligned}
\int_{1}^{x} \lambda^{-2 \varrho} & W_{1}(y) d y=\int_{1}^{x} \lambda^{-2 \varrho} \sum_{n \geq 1} \frac{\left|a_{n}\right|^{2}\left|\Delta_{\lambda}^{\varrho} I_{\varrho}\left(\alpha_{n} y\right)\right|^{2}}{\alpha_{n}^{2(1+\varrho)}} d y \\
= & \int_{1}^{x} \lambda^{-2 \varrho} \sum_{n \geq 1} \frac{\left|a_{n}\right|^{2}\left(\lambda^{\varrho} \alpha_{n}^{\varrho}\left(\alpha_{n} y\right)^{(1-\delta) / 2}\right)^{2}}{\alpha_{n}^{2(1+\varrho)}} d y \ll \int_{1}^{x} \sum_{n \geq 1} \frac{\left|a_{n}\right|^{2}}{\alpha_{n}^{1+\delta}} y^{1-\delta} d y .
\end{aligned}
$$

For all $\varepsilon>0, \sum_{\alpha_{n} \leq x}\left|a_{n}\right|^{2}=O_{\varepsilon}\left(x^{1+\varepsilon}\right)$, so if $\varepsilon<\delta$, then $\sum_{n \geq 1}\left|a_{n}\right|^{2} / \alpha_{n}^{1+\delta}$ $=O_{\delta}(1)$. This proves

$$
\int_{1}^{x} \sum_{n \geq 1} \frac{\left|a_{n}\right|^{2}}{\alpha_{n}^{1+\delta}} y^{1-\delta} d y=O_{\delta}\left(x^{2-\delta}\right)
$$

\subsubsection{Estimation of non-diagonal terms}

Notation. We recall that

$$
\omega=\left(\varrho+\frac{1}{2}\right)(1-\delta)-i \delta \Im \xi, \quad \alpha_{n}=n / Q^{2}, \quad a=d \beta^{\delta} .
$$

Equation (27) shows that the estimation of the non-diagonal terms reduces to estimating the integral

$$
\int_{1}^{x} \lambda(y)^{-2 \varrho} \Delta_{\lambda}^{\varrho} I_{\varrho}\left(\alpha_{m} y\right) \Delta_{\lambda}^{\varrho} \bar{I}_{\varrho}\left(\alpha_{n} y\right) d y .
$$

In view of the asymptotic expansion of $I_{\varrho}$ given in Theorem 7.1, this estimate is reduced to that of the integral

$$
U_{m, n}^{(b, c)}(x)=\int_{1}^{x} \lambda(y)^{-2 \varrho} \Delta_{\lambda}^{\varrho}\left(y^{\omega} e^{i b y^{\delta} \alpha_{m}^{\delta}}\right) \Delta_{\lambda}^{\varrho}\left(y^{\bar{\omega}} e^{-i c y^{\delta} \alpha_{n}^{\delta}}\right) d y,
$$

where $b$ and $c$ are real numbers such that $|b|=|c|=a$.

Lemma 9.1. For $\delta<1, \varrho \geq 1, z>1$ and $x>\max \left(1,(2 \varrho)^{1 /(\eta+\delta)}\right)$, we have

$$
\begin{aligned}
& U_{m, n}^{b, c}(x)=O\left(\frac{1}{\alpha_{m}^{\delta}-\alpha_{n}^{\delta}}\right) x^{2(1-\delta)}\left(\alpha_{m} \alpha_{n}\right)^{\delta \varrho} \quad \text { if } \alpha_{n}<\alpha_{m}<z \\
& U_{m, n}^{b, c}(x)=O\left(\frac{\alpha_{n}^{\delta \varrho}}{\alpha_{m}^{\delta}-\alpha_{n}^{\delta}}\right) x^{(\varrho+2)(1-\delta)}\left(1+\lambda x^{\delta-1} \alpha_{m}^{\delta}\right) \\
& \text { if } \alpha_{n} \leq z<\alpha_{m}
\end{aligned}
$$




$$
\begin{aligned}
U_{m, n}^{b, c}(x)=O\left(\frac{1}{\alpha_{m}^{\delta}-\alpha_{n}^{\delta}}\right) \lambda^{-2 \varrho} x^{(2 \varrho+1)(1-\delta)}\left(x^{1-\delta}+\lambda \alpha_{m}^{\delta}\right) & \\
& \text { if } z<\alpha_{n}<\alpha_{m} .
\end{aligned}
$$

Proof. In this proof, $m$ and $n$ are fixed so we do not always write them. For $b, c \in \mathbb{R} \backslash\{0\}$ such that $|b|=|c|$ and for $y>0$, we define

$$
G_{m}^{b}(y)=e^{-i b y^{\delta} \alpha_{m}^{\delta}} \Delta_{\lambda}^{\varrho}\left(y^{\omega} e^{i b y^{\delta} \alpha_{m}^{\delta}}\right), \quad H^{b, c}(y)=\lambda(y)^{-2 \varrho} y^{1-\delta} G_{m}^{b}(y) \overline{G_{n}^{c}(y)},
$$

so we have

$$
\begin{aligned}
U_{m, n}^{b, c}(x) & =\int_{1}^{x} \lambda(y)^{-2 \varrho} G_{m}^{b}(y) \overline{G_{n}^{c}(y)} e^{i y^{\delta}\left(b \alpha_{m}^{\delta}-c \alpha_{n}^{\delta}\right)} d y \\
& =\frac{1}{i \delta\left(b \alpha_{m}^{\delta}-c \alpha_{n}^{\delta}\right)} \int_{1}^{x} H^{b, c}(y) \frac{d}{d y}\left(e^{i y^{\delta}\left(b \alpha_{m}^{\delta}-c \alpha_{n}^{\delta}\right)}\right) d y
\end{aligned}
$$

and hence

$$
\begin{aligned}
& U_{m, n}^{b, c}(x) \\
& \quad=O\left(\frac{1}{\left|\alpha_{m}^{\delta}-\alpha_{n}^{\delta}\right|}\right)\left(\left|H^{b, c}(x)\right|+\left|H^{b, c}(1)\right|+\int_{1}^{x}\left|\frac{d}{d y} H^{b, c}(y)\right| d y\right) .
\end{aligned}
$$

We have

$$
\begin{aligned}
\left|G_{m}^{b}(y)\right| & =\left|\Delta_{\lambda}^{r}\left(e^{i b y^{\delta} \alpha_{m}^{\delta}} y^{\omega}\right)\right| \\
\left|\frac{d}{d y} G_{m}^{b}(y)\right| & =\left|e^{i b \alpha_{m}^{\delta} y^{\delta}} \frac{d}{d y}\left(e^{-i b \alpha_{m}^{\delta} y^{\delta}} \Delta_{\lambda}^{\varrho}\left(y^{\omega} e^{i b \alpha_{m}^{\delta} y^{\delta}}\right)\right)\right|
\end{aligned}
$$

and these two functions have been estimated by K. Chandrasekharan and R. Narasimhan in [CN2] in case $\omega$ is real. In case $\omega \in \mathbb{C}$, the estimates and their proofs are the same (see $[\mathrm{dR} 1]$ for more details). We recall here their results:

If $y>\max \left(1,(2 \varrho)^{1 /(\eta+\delta)}\right)$, we have

$$
\begin{aligned}
& G_{m}^{b}(y)=O\left(y^{\Re \omega}\right), \\
& G_{m}^{b}(y)=y^{\omega} \Delta_{\lambda}^{\varrho}\left(e^{i b \alpha_{m}^{\delta} y^{\delta}}\right)+O\left(\lambda y^{\Re \omega-1}\right), \\
& G_{m}^{b}(y)=O\left(\lambda^{\varrho} y^{\Re \omega-\varrho(1-\delta)} \alpha_{m}^{\delta \varrho}\right),
\end{aligned}
$$

and

$$
\begin{aligned}
& \frac{d}{d y} G_{m}^{b}(y)=O\left(\lambda \alpha_{m}^{\delta} y^{\delta-2+\Re \omega}\right) \quad \text { for } \varrho \geq 1 \\
& \frac{d}{d y} G_{m}^{b}(y)=O\left(\lambda^{\varrho} y^{\Re \omega-\varrho(1-\delta)-1} \alpha_{m}^{\delta \varrho}\right) .
\end{aligned}
$$


Using $\lambda^{\prime}(y)=O(\lambda(y) / y)$, we get

$$
\begin{aligned}
&\left|\frac{d}{d y} H^{b, c}(y)\right|=O\left(y^{-1} H^{b, c}(y)\right) \\
&+\lambda(y)^{-2 \varrho} y^{1-\delta} O\left(\left|\frac{d}{d y} G_{m}^{b}(y)\right|\left|G_{n}^{c}(y)\right|+\left|G_{m}^{b}(y)\right|\left|\frac{d}{d y} G_{n}^{c}(y)\right|\right) .
\end{aligned}
$$

If $\alpha_{n}<\alpha_{m} \leq z$, by (35) and (37) we have

$$
\begin{aligned}
H_{k, l}^{b, c}(y) & =\lambda^{-2 \varrho} y^{1-\delta} O\left(\lambda^{2 \varrho} y^{2 \Re \omega-2 \varrho(1-\delta)}\left(\alpha_{m} \alpha_{n}\right)^{\delta \varrho}\right) \\
& =O\left(y^{2(1-\delta)}\left(\alpha_{m} \alpha_{n}\right)^{\delta \varrho}\right)
\end{aligned}
$$

and by (38),

$$
\begin{aligned}
\frac{d}{d y} H^{b, c}(y) & =O\left(\lambda^{-2 \varrho} y^{-\delta} \lambda^{2 \varrho} y^{2 \Re \omega-2 \varrho(1-\delta)}\left(\alpha_{m} \alpha_{n}\right)^{\delta \varrho}\right) \\
& =O\left(y^{1-2 \delta}\left(\alpha_{m} \alpha_{n}\right)^{\delta \varrho}\right) .
\end{aligned}
$$

By (39), (40) and (32), we get (29).

If $\alpha_{n} \leq z<\alpha_{m}$, by (33) applied to $G_{m}^{b}(y)$, (35) to $G_{n}^{c}(y)$, (36) to $\frac{d}{d y} G_{m}^{b}(y)$ and $(37)$ to $\frac{d}{d y} G_{n}^{c}(y)$, we have

$$
H^{b, c}(y)=\lambda^{-2 \varrho} y^{1-\delta} O\left(\lambda^{\varrho} y^{2 \Re \omega-\varrho(1-\delta)} \alpha_{n}^{\delta \varrho}\right)=O\left(\lambda^{-\varrho} y^{(\varrho+2)(1-\delta)} \alpha_{n}^{\delta \varrho}\right),
$$

and by (38),

$$
\begin{aligned}
& \frac{d}{d y} H^{b, c}(y)=O\left(\lambda^{-\varrho} y^{2 \Re \omega-(\varrho-1)(1-\delta)-1} \alpha_{n}^{\delta \varrho}\right) \\
+ & \lambda^{-2 \varrho} y^{1-\delta} O\left(\lambda y^{\Re \omega-2+\delta} \alpha_{m}^{\delta} \lambda^{\varrho} y^{\Re \omega-\varrho(1-\delta)} \alpha_{n}^{\delta \varrho}+y^{\Re \omega} \lambda^{\varrho} y^{\Re \omega-1-\varrho(1-\delta)} \alpha_{n}^{\varrho \delta}\right) \\
= & O\left(\lambda^{-\varrho} y^{-\delta+2 \Re \omega-\varrho(1-\delta)} \alpha_{n}^{\delta \varrho}\right)+O\left(\lambda^{-\varrho+1} y^{2 \Re \omega-\varrho(1-\delta)-1} \alpha_{m}^{\delta} \alpha_{n}^{\delta \varrho}\right) \\
= & O\left(\lambda^{-\varrho} y^{(\varrho+1)(1-\delta)-\delta} \alpha_{n}^{\delta \varrho}\left(1+\lambda y^{\delta-1} \alpha_{m}^{\delta}\right)\right) .
\end{aligned}
$$

By (41), (42) and (32), we get (30).

If $z<\alpha_{n}<\alpha_{m}$, by (33) and (36), we have

$$
\left|H^{b, c}(y)\right|=\lambda^{-2 \varrho} y^{1-\delta} O\left(y^{2 \Re \omega}\right)=O\left(\lambda^{-2 \varrho} y^{(2 \varrho+2)(1-\delta)}\right),
$$

and by (38),

$$
\frac{d}{d y} H^{b, c}(y)=O\left(\lambda^{-2 \varrho} y^{2 \Re \omega-\delta}\right)+\lambda^{-2 \varrho+1} y^{2 \Re \omega-1} O\left(\alpha_{m}^{\delta}+\alpha_{n}^{\delta}\right),
$$

so

$$
\frac{d}{d y} H^{b, c}(y)=O\left(\lambda^{-2 \varrho} y^{(2 \varrho+1)(1-\delta)-\delta}\left(1+\lambda y^{\delta-1} \alpha_{n}^{\delta}\right)\right) .
$$

By (43), (44) and (32), we have (31). 
We can deduce from these estimates those of non-diagonal terms. The proof is the same as in [CN2], we just recall here their conclusions.

Lemma 9.2. Assume that

$$
\begin{gathered}
W_{2}(x)=\sum_{m \neq n} \frac{b_{m} \bar{b}_{n}}{\left(\alpha_{m} \alpha_{n}\right)^{1+\varrho}} \Delta_{\lambda}^{\varrho} I_{\varrho}\left(\alpha_{m} y\right) \Delta_{\lambda}^{\varrho} \bar{I}_{\varrho}\left(\alpha_{n} y\right), \\
I_{\varrho}(x) \sim e^{i a x^{\delta}} \sum_{\nu=0}^{M} \delta_{\nu} x^{\omega-\nu \delta}+e^{-i a x^{\delta}} \sum_{\nu=0}^{M} \delta_{\nu}^{\prime} x^{\omega-\nu \delta},
\end{gathered}
$$

and the estimates of Lemma 9.1 hold for the function

$$
U_{m, n}^{(b, c)}(x)=\int_{1}^{x} \lambda(y)^{-2 \varrho} \Delta_{\lambda}^{\varrho}\left(y^{\omega} e^{i b y^{\delta} \alpha_{m}^{\delta}}\right) \Delta_{\lambda}^{\varrho}\left(y^{\bar{\omega}} e^{-i c y^{\delta} \alpha_{n}^{\delta}}\right) d y,
$$

for all $b, c \in \mathbb{R}$ such that $|b|=|c|=a$. Then for all $\varepsilon>0$, we have

$$
\int_{1}^{x} \lambda^{-2 \varrho} W_{2}(y) d y=O\left(x^{2(1-\delta)}\left(x^{\eta(d-2)+\varepsilon}+\log x\right)\right) .
$$

9.3. Proof of Theorem 2.2. Under the hypothesis of Theorem 2.2, we have

$$
\int_{1}^{x} \lambda^{-2 \varrho}|W(y)|^{2} d y=O\left(x^{2-\delta}\right)+O\left(x^{2(1-\delta)}\left(x^{\eta(d-2)+\varepsilon}+\log x\right)\right)
$$

and

$$
\int_{1}^{x}|V(y)|^{2} d y=O\left(x^{3-2 \delta-2 \eta+\varepsilon}\right) .
$$

Finally, for all $\eta>0$, we have

$$
\int_{1}^{x}|E(y)|^{2} d y=O\left(x^{2-\delta}\right)+O\left(x^{2(1-\delta)}\left(x^{\eta(d-2)+\varepsilon}+\log x\right)\right)+O\left(x^{3-2 \delta-2 \eta+\varepsilon}\right) .
$$

If $d<2$, then we let $\eta \rightarrow \infty$ to obtain

$$
\int_{1}^{x}|E(y)|^{2} d y=O\left(x^{2-\delta}\right) .
$$

If $d \geq 2$, then we choose $\eta=\delta$ to get

$$
\int_{1}^{x}|E(y)|^{2} d y=O\left(x^{2-\delta}\right)+O\left(x^{3-4 \delta+\varepsilon}\right) .
$$

This ends the proof.

Estimates of Theorem 2.2 prove that for $d<4$, the function $E(x)$ belongs to $L^{2}\left(\left[0, \infty\left[, d x / x^{2}\right)\right.\right.$. We use this in $[\mathrm{dR} 2]$. The estimate we get in this last 
theorem is similar to the one obtained by Chandrasekharan and Narasimhan in [CN2]. We intend to adapt the method used by Redmond in [R2] in order to sharpen our result in the case $d \geq 4$ and avoid the use of the hypothesis $\sum_{n \leq x}\left|a_{n}\right|^{2} \ll x^{1+\varepsilon}$.

\section{References}

[Bo] N. Bourbaki, Éléments de mathématiques. Fonctions d'une variable réelle. Théorie élémentaire, Hermann, 1976.

[Br] B. L. J. Braaksma, Asymptotic expansions and analytic continuations for a class of Barnes-integrals, Compos. Math. 15 (1964), 239-341.

[Ch] K. Chandrasekharan, Arithmetical Functions, Grundlehren Math. Wiss. 167, Springer, 1970.

[CN1] K. Chandrasekharan and R. Narasimhan, Functional equations with multiple gamma factors and the average order of arithmetical functions, Ann. of Math. 76 (1962), 93-136.

[CN2] - - - On the mean value of the error term for a class of arithmetical functions, Acta Math. 112 (1963), 41-67.

[CN3] - - - The approximate functional equation for a class of zeta functions, Math. Ann. 152 (1963), 30-64.

[CG1] J. B. Conrey and A. Ghosh, On the Selberg class of Dirichlet series: small degrees, Duke Math. J. 72 (1993), 673-693.

[CG2] -, 一, Remarks on the generalised Lindelöf hypothesis, preprint.

[Cr] H. Cramér, Über zwei Sätze des Herrn G. H. Hardy, Math. Z. 15 (1922), 201-210.

[KP0] J. Kaczorowski and A. Perelli, The Selberg class: a survey, in: Number Theory in Progress, Vol. 2 (Zakopane-Kościelisko, 1997), de Gruyter, Berlin, 1999, 953-992.

[KP1] - - - On the structure of the Selberg class, I: $0 \leq d \leq 1$, Acta Math. 182 (1999), $207-241$.

[KP2] - - - On the structure of the Selberg class, $V: 1<d<5 / 3$, Invent. Math. 150 (2002), 485-516.

[Lan] E. Landau, Über die Anzahl der Gitterpunkte in gewissen Bereichen, Gött. Nachr. $1912,687-771$.

[M] M. R. Murty, Selberg's conjectures and Artin L-functions, Bull. Amer. Math. Soc. 31 (1994), 1-14.

[R1] D. Redmond, An O theorem for a class of Dirichlet series, Math. J. Okayama Univ. 28 (1986), 151-158.

[R2] -, Mean value theorems for a class of Dirichlet series, Pacific J. Math. 78 (1978), $191-231$.

[dR1] A. de Roton, Généralisation du critère de Beurling-Nyman à la classe de Selberg, thèse, Univ. Bordeaux 1, 2003.

[dR2] - Généralisation du critère de Beurling et Nyman pour l'hypothèse de Riemann, Trans. Amer. Math. Soc., to appear.

[S] A. Selberg, Old and new conjectures and results about a class of Dirichlet series, in: Collected Papers, Volume II, 47-63.

[T] G. Tenenbaum, Introduction à la théorie analytique et probabiliste des nombres, Société Math. de France, 1995.

[V] G. Voronoï, Sur une fonction transcendante et ses applications à la sommation de quelques séries, Ann. École Norm. Sup. (3) 21 (1904), 207-267, 459-533. 
[W] G. N. Watson, A Treatise on the Theory of Bessel Functions, 2nd ed., Cambridge Univ. Press, 1966.

IECN-Université Henri Poincaré, Nancy 1

BP 239

F-54506 Vandœuvre-lès-Nancy Cedex, France

E-mail: deroton@iecn.u-nancy.fr

Received on 1.4.2004

and in revised form on 24.10.2006 